\title{
Estudo de viabilidade para aproveitamento de água pluvial para fins não potáveis no ITPAC, em Porto Nacional/TO
}

A água é um recurso fundamental à vida e sua disponibilidade depende da preservação ambiental. O Brasil possui uma grande disponibilidade hídrica, porém algumas regiões já se encontram com escassez de água. Diante disso, surgem alternativas para evitar o desperdício, entre elas o aproveitamento de água das águas pluviais, que capta e armazena a água da chuva para utilizar em fins não potáveis, como descargas, irrigações de jardim, entre outras. $O$ método é fundamental para a implantação em universidades, visto que, em locais onde o consumidor não é responsabilizado diretamente pelo consumo de água, tende a haver um desperdício maior. Nesse sentido, a presente pesquisa buscou realizar um estudo de viabilidade para implantação de um sistema de aproveitamento de água em um bloco do Instituto Tocantinense Presidente Antônio Carlos - ITPAC, identificando o reservatório necessário a partir do Método de Azevedo Neto, previsto na ABNT NBR 15527/2007. A partir da pesquisa, identificou-se que a cidade de Porto Nacional possui um índice pluviométrico variável mensalmente, com maiores índices entre os meses de outubro de abril, havendo uma seca principalmente no mês de julho. Apesar do período de seca verificado, ele não possui um grande impacto negativo para a implantação do projeto, visto que se trata de um período de férias, onde a instituição tem pouca atividade. Além disso, identificou-se que a instituição dispõe de meios onde não faz o uso da água da rede de abastecimento pública, entretanto a implantação do método é viável, com viabilidade econômica e ambiental, e pode servir de um método de estudo para os acadêmicos de Engenharia Civil e Arquitetura e Urbanismo da instituição.

Palavras-chave: Águas pluviais; Aproveitamento; Reuso; Viabilidade.

\section{Feasibility study for the use of pluvial water for non-potable purposes in ITPAC in Porto Nacional/TO}

Water is a fundamental resource for life and its availability depends on environmental preservation. Brazil has a large water availability, but some regions are already short of water. Thus, alternatives to avoid waste arise, including the use of rainwater, which captures and stores rainwater for use in non-potable purposes, such as discharges, garden irrigation, among others. The method is fundamental for implementation in universities, since in places where the consumer is not directly responsible for water consumption, there tends to be a greater waste. In this sense, this research aimed to conduct a feasibility study for the implementation of a water use system in a block of the Instituto Tocantinense Presidente Antônio Carlos - ITPAC, identifying the necessary reservoir from the Azevedo Neto Method, provided for in ABNT NBR 15527 / 2007. From the research, it was identified that the city of Porto Nacional has a monthly variable rainfall index, with higher rates between the months of October April, with a drought mainly in July. Despite the period of drought, it does not have a major negative impact on the implementation of the project, as it is a vacation, where the institution has little activity. In addition, it was identified that the institution has means where it does not make use of water from the public supply network, however the implementation of the method is feasible, with economic and environmental viability, and may serve as a study method for academics of Civil Engineering and Architecture and Urbanism of the institution.

Keywords: Rainwater; Use; Reuse; Viability.

Topic: Engenharia Civil

Reviewed anonymously in the process of blind peer
Received: $12 / 03 / 2019$

Approved: 23/07/2019
Jessica Priscilla Silva Pereira

Instituto Tocantinense Presidente Antônio Carlos, Brasil http://lattes.cnpq.br/1577440409383566

jessicapriscilla.jp@gmail.com

Alesi Teixeira Mendes $(i b)$

Instituto Tocantinense Presidente Antônio Carlos, Brasil

http://lattes.cnpq.br/2473113080999082

http://orcid.org/0000-0002-5632-7235

alesitmendes@gmail.com
Referencing this:

PEREIRA, J. P. S.; MENDES, A. T.. Estudo de viabilidade para aproveitamento de água pluvial para fins não potáveis no ITPAC, em Porto Nacional/TO. Inventionis, v.1, n.2, p.1-8, 2019. DOI: http://doi.org/10.6008/CBPC2674-6395.2019.002.0001

DOI: 10.6008/CBPC2674-6395.2019.002.0001

${ }^{\circledR}$ Companhia Brasileira de Produção Científica. All rights reserved. 


\section{INTRODUÇÃO}

A água é um recurso essencial à vida, e importante para que haja desenvolvimento econômico e tecnológico, sendo encontrada em grande quantidade no planeta, porém somente cerca de $0,007 \%$ de toda a água é encontrada facilmente para o consumo humano, e apesar o pequeno valor, é suficiente para abastecer as populações (MARINOSKI, 2007).

Apesar de haver bastante água no planeta Terra, ela é desigualmente distribuída, no Brasil, por exemplo, quase $70 \%$ da água doce se encontra em apenas uma região, além disso, com o crescimento da população mundial, a poluição e o desperdício, o ciclo hidrológico não é suficiente para abastecer a todos, e alguns lugares acabam sofrendo com a escassez da água (SODRÉ, 2018; MARINOSKI, 2007).

De acordo com Schneider et al. (2016), diante da escassez de água, há uma necessidade e preocupação em encontrar meios para usufruir dos recursos hídricos com menos desperdícios e mais consciência. Segundo Azevêdo (2016), é necessário a implantação de métodos, mudanças e aperfeiçoamento no sistema de gestão de água, com a utilização de alternativas para o uso racional e conservação da água, pois podem contribuir para a diminuição dos problemas hídricos da atualidade e os futuros.

Diante desse contexto, surge a alternativa de aproveitamento da água pluvial para o consumo não potável em edificações, que contribui para a diminuição da demanda de água fornecida, diminuição dos custos com água potável, diminuição do desperdício de água potável (MAY, 2004). A prática de aproveitamento de água das chuvas para fins não potáveis é uma proposta pouco convencional para a conservação de água no planeta, e é utilizada para fins menos exigentes da utilização da água, como: descargas, irrigação de jardins, lavagem de calçadas, lavagem de carros etc. (NAZÁRIO et al., 2013).

Segundo a NBR 15527/2007, as águas das chuvas podem ser utilizadas após passar por um tratamento adequado, nas atividades menos exigentes, e para isso deve haver um sistema para captar e reservar as águas pluviais, onde a determinação do volume do reservatório pode ser por seis métodos: de Rippl, da simulação, Azevedo Neto, prático alemão, prático inglês e prático australiano.

Dentre os muitos locais onde pode-se implantar métodos para reaproveitamento de água da chuva, pode-se destacar as universidades. Segundo Azevêdo (2016) a redução do consumo de água nos prédios de instituições é o primeiro passo para diminuir a demanda e contribuir para a conscientização das pessoas para minimizar os impactos da escassez de água. A importância é significativa em virtude de que em universidades, o uso da água possui muitos desperdícios, pois o usuário não é o responsável direto para o pagamento da conta de abastecimento de água (MARINOSKI, 2007). Diante do exposto, o presente trabalho objetivou avaliar a implantação de um projeto para reaproveitamento pluvial para fins não potáveis no Instituto Tocantinense Presidente Antônio Carlos, através do uso do método Azevedo Neto.

\section{METODOLOGIA}

\section{Tipo de Pesquisa}

A pesquisa é caracterizada como do tipo aplicada, explicativa, descritiva, estudo de caso e 
bibliográfica, através de uma abordagem quantitativa.

\section{Caracterização do Local da Pesquisa}

A pesquisa foi realizada na cidade de Porto Nacional - TO, localizada no estado do Tocantins, norte do país. Uma região marcada por longos períodos de estiagem. Especificamente, foi realizada no Instituto Tocantinense Presidente Antônio Carlos - ITPAC Porto, localizada no setor Jardim dos Ypês, da cidade de Porto Nacional - TO, conforme a figura 1. A instituição é particular, com cursos de graduação e pósgraduação, comportando alunos dos cursos de Engenharia Civil, Medicina, Enfermagem, Odontologia, Administração, Arquitetura e Urbanismo, Agronomia e Agronegócio. Por esse motivo, possui uma área muito extensa, conforme a planta baixa apresentada na figura 2 .

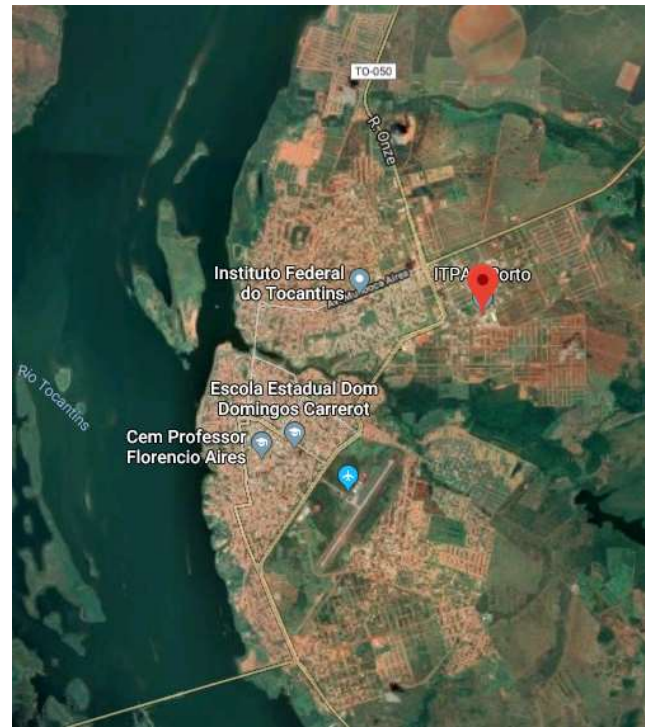

Figura 2: Localização do ITPAC Porto.

Fonte: Google Maps (2019).

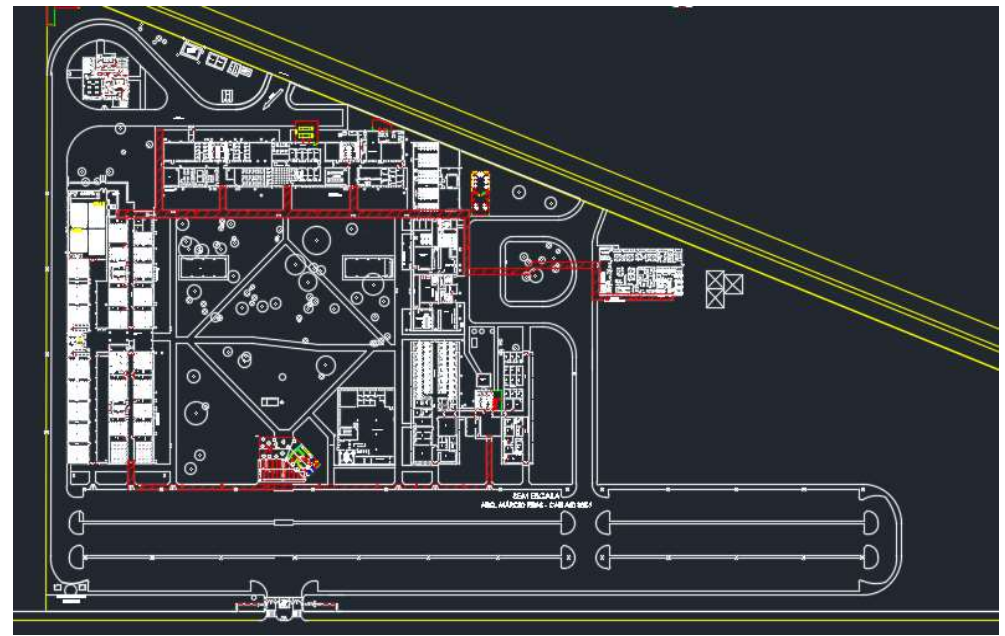

Figura 1: Planta baixa do ITPAC Porto.

\section{Procedimentos Metodológicos}

Após a apresentação e aprovação do projeto de pesquisa, procurou-se a instituição para autorizar a realização da pesquisa, bem como para disponibilizarem os dados referentes ao consumo de água. Utilizouse de trabalhos afins realizados na mesma instituição para coletar os dados também. Além dos dados do ITPAC, coletou-se dados referentes ao índice de chuvas da cidade de Porto Nacional - TO, a partir do Instituto Nacional de Metereologia - INMET, do Ministério da Agricultura, Pecuária e Abastecimento. Os dados foram coletados durante os meses de setembro, outubro e novembro de 2019.

Além do índice de chuvas, coletou-se os dados do maior número de dias de seca por mês, durante os últimos 10 anos, de 2009 a 2018. Os dados de 2019 também foram coletados, entretanto não há uma finalização para os meses de novembro e dezembro. Para a coleta dos dias de seca, utilizou-se o gráfico 'chuva acumulada em $24 \mathrm{~h}$ ' por cada mês, disponibilizado pelo INMET, verificando quais dias não tiveram chuva e contabilizando a maior quantidade de dias consecutivos sem chuva por mês. Após a coleta desses dados, realizou-se o dimensionamento dos reservatórios para água da chuva, de acordo com o método Azevedo Neto, da NBR 15527:2007. Ele prevê: 


\section{$V=0,042 \times P \times A \times T$}

Onde: $V$ é o valor do volume de água que pode ser aproveitável e o volume de água do reservatório, em litros; P se refere ao valor da precipitação média anual de chuva, em milímetros; A é o valor da área de coleta em projeção, em metros quadrados; T é o valor do número de meses de seca ou de pouca chuva.

A análise dos dados consistiu principalmente visando a viabilidade do ITPAC na implantação de um sistema de coleta e armazenagem de água a partir da verificação do volume do reservatório. Não se fez necessário a realização de ensaios laboratoriais, em virtude de a pesquisa ser um estudo de viabilidade.

\section{RESULTADOS E DISCUSSÃO}

Ao analisar a viabilidade do ITPAC Porto para a implantação de um sistema de coleta de águas pluviais, a instituição forneceu dados que a água utilizada não é proveniente do abastecimento urbano de água pela empresa fornecedora. Isso porque a instituição detém de poço artesiano, além de armazenar água a partir da compra por caminhões pipas, tornando-se mais barato o consumo, visto que, ao considerar a quantidade de cursos e dos funcionários necessários para o funcionamento na instituição, acredita-se haver mais de 2 mil pessoas que utilizam o prédio, o que por consequência há um alto consumo de água.

Apesar disso, a implantação de um sistema de coleta e armazenagem de água da chuva se apresenta como uma alternativa sustentável a ser adotada. Ao investigar a incidência de chuvas no município de Porto Nacional - TO, nos últimos 10 anos, entre 2009 e 2018, a partir dos gráficos disponibilizados pelo INMET, identificou-se que o índice pluviométrico é variável anualmente e mensalmente, conforme o quaro 1.

Quadro 1: Índice pluviométrico em Porto Nacional nos últimos 10 anos.

\begin{tabular}{|c|c|c|c|c|c|c|c|c|c|c|c|}
\hline \multirow{3}{*}{$\stackrel{y}{\tilde{y}}$} & \multicolumn{11}{|l|}{ Anos } \\
\hline & ஜํ. & 울 & नें & జ & $\stackrel{m}{\stackrel{n}{n}}$ & d্ & นึ้ & 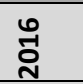 & नें & $\stackrel{\infty}{a}$ & $\begin{array}{l}\text { TOTAL } \\
\text { em mm }\end{array}$ \\
\hline & \multicolumn{11}{|c|}{ Índice Pluviométrico em mm* } \\
\hline Jan & 100 & 298 & 351 & 333 & 348 & 226 & 175 & 460 & 176 & 138 & 2605 \\
\hline Fev & 265 & 200 & 325 & 375 & 255 & 305 & 205 & 36 & 165 & 335 & 2466 \\
\hline Mar & 220 & 425 & 342 & 150 & 395 & 230 & 273 & 178 & 150 & 220 & 2583 \\
\hline Abr & 200 & 140 & 200 & 150 & 100 & 100 & 277 & 40 & 85 & 336 & 1628 \\
\hline Mai & 187 & 55 & 22 & 80 & 50 & 40 & 38 & 15 & 22 & 7 & 516 \\
\hline Jun & 12 & 5 & 0 & 20 & 8 & 0 & 0 & 10 & 0 & 0 & 55 \\
\hline Jul & 0 & 0 & 0 & 0 & 0 & 0 & 4 & 0 & 0 & 0 & 4 \\
\hline Ago & 5 & 0 & 0 & 0 & 15 & 0 & 0 & 8 & 0 & 16 & 44 \\
\hline Set & 50 & 0 & 0 & 4 & 13 & 75 & 15 & 37 & 0 & 10 & 204 \\
\hline Out & 251 & 80 & 130 & 75 & 100 & 50 & 56 & 140 & 42 & 179 & 1103 \\
\hline Nov & 320 & 90 & 195 & 243 & 188 & 251 & 65 & 198 & 193 & 242 & 1985 \\
\hline Dez & 280 & 200 & 240 & 80 & 505 & 325 & 112 & 292 & 260 & 239 & 2533 \\
\hline TOTAL em mm & 1890 & 1493 & 1805 & 1510 & 1977 & 1602 & 1220 & 1414 & 1093 & 1722 & 15726 \\
\hline
\end{tabular}

* Dados do INMET.

Observa-se que não há um valor médio padrão anualmente, pelo contrário, há uma variação constante, sendo que o ano com o maior índice pluviométrico foi 2010, com 1890mm, e o menor foi 2017, com 1093mm, havendo uma diferença muito grande entre ambos. A partir dos dados do quadro 1, é possível identificar a média pluviométrica mensal e total para os 10 anos analisados, conforme a tabela 1.

Tabela 1: Média Pluviométrica em Porto Nacional - TO.

\begin{tabular}{|l|l|}
\hline \multicolumn{1}{|c|}{ MESES } & \multicolumn{1}{c|}{ PRECIPITAÇÃO MÉDIA (MM) } \\
\hline JANEIRO & $260,5 \mathrm{~mm}$ \\
\hline FEVEREIRO & $246,6 \mathrm{~mm}$ \\
\hline
\end{tabular}




\begin{tabular}{|c|c|}
\hline MARÇO & $258,3 \mathrm{~mm}$ \\
\hline ABRIL & $162,8 \mathrm{~mm}$ \\
\hline MAIO & $51,6 \mathrm{~mm}$ \\
\hline JUNHO & $5,5 \mathrm{~mm}$ \\
\hline JULHO & $0,4 \mathrm{~mm}$ \\
\hline AGOSTO & $4,4 \mathrm{~mm}$ \\
\hline SETEMBRO & $20,4 \mathrm{~mm}$ \\
\hline OUTUBRO & $110,3 \mathrm{~mm}$ \\
\hline NOVEMBRO & $198,5 \mathrm{~mm}$ \\
\hline DEZEMBRO & $253,3 \mathrm{~mm}$ \\
\hline MÉDIA ANUAL & $1572,6 \mathrm{~mm}$ \\
\hline
\end{tabular}

Identifica-se que os meses de maior índice pluviométrico são, respectivamente: janeiro, março, dezembro e fevereiro, com índices acima de $200 \mathrm{~mm}$. Os meses de novembro, abril e outubro possuem índices medianos, acima de $100 \mathrm{~mm}$. Somente os meses compreendidos entre o meio do ano são períodos de seca, entre maio e setembro, sendo que julho é o período mais crítica, com menos de $1 \mathrm{~mm}$, além de junho e agosto, com índices abaixo de $10 \mathrm{~mm}$.

O período mais crítico compreende o mês de férias no Instituto Tocantinense Presidente Antônio Carlos, desse modo, identifica-se que o uso de águas pluviais para fins não potáveis é satisfatório para o ITPAC Porto, visto que os períodos de aula compreendem meses onde há bons índices pluviométricos. Ao considerar o período acadêmico disposto nos calendários do ITPAC, identifica-se que as aulas costumam variar, para o primeiro semestre, entre a última semana de janeiro e o meio de junho, e o segundo semestre entre a primeira semana de agosto e o meio de dezembro. Nesse sentido, ao considerar os meses de aula, identifica-se que o ITPAC enfrenta períodos de seca que inviabilizam o uso de águas pluviais para fins não potáveis somente durante todo os meses de maio, agosto e setembro, e parte do mês de junho. Entretanto os meses mais críticos são junho e agosto.

Quadro 2: Número máximo de dias sem chuva em Porto Nacional de 2009 a 2018.

\begin{tabular}{|c|c|c|c|c|c|c|c|c|c|c|}
\hline \multirow{3}{*}{ Mês } & \multicolumn{10}{|c|}{ Número máximo de dias sem chuva* } \\
\hline & \multicolumn{10}{|l|}{ Ano } \\
\hline & 2009 & 2010 & 2011 & 2012 & 2013 & 2014 & 2015 & 2016 & 2017 & 2018 \\
\hline Jan & 4 & 5 & 3 & 2 & 5 & 4 & 4 & 3 & 6 & 6 \\
\hline Fev & 3 & 4 & 2 & 5 & 8 & 1 & 4 & 10 & 2 & 1 \\
\hline Mar & 3 & 2 & 2 & 4 & 4 & 1 & 3 & 3 & 4 & 4 \\
\hline Abr & 4 & 12 & 5 & 6 & 6 & 5 & 4 & 14 & 4 & 5 \\
\hline Mai & 10 & 8 & 17 & 12 & 15 & 11 & 10 & 19 & 11 & 15 \\
\hline Jun & 27 & 27 & 30 & 25 & 24 & 30 & 30 & 18 & 30 & 30 \\
\hline Jul & 31 & 17 & 31 & 31 & 31 & 31 & 24 & 31 & 31 & 31 \\
\hline Ago & 24 & 31 & 31 & 31 & 29 & 30 & 31 & 24 & 31 & 16 \\
\hline Set & 10 & \begin{tabular}{|l|}
30 \\
\end{tabular} & 30 & 21 & 10 & 16 & 29 & 17 & 30 & 21 \\
\hline Out & 3 & 5 & 3 & 9 & 7 & 10 & 13 & 6 & 18 & 4 \\
\hline Nov & \begin{tabular}{|l|}
2 \\
\end{tabular} & 5 & 3 & 4 & 3 & 3 & 5 & 5 & 3 & 5 \\
\hline Dez & 3 & 4 & 4 & 5 & 3 & 2 & 4 & 5 & 5 & 13 \\
\hline
\end{tabular}

* Dados do INMET.

Em virtude da aplicação do Método de Azevedo Neto para o cálculo do reservatório, a identificação dos dias sem chuva se refere ao principal dado a ser identificado (GIACCHINI et al., 2011). Nesse sentido, a partir de dados do INMET, identificou-se o número máximo de dias sem chuva por mês e ano conforme o quadro 2. A partir dos dados do quadro 2, é possível obter a média do número máximo de dias sem chuva por mês de 2009 a 2018, conforme a tabela 2 e figura 3. 
Tabela 2: Média mensal do número máximo de dias sem chuva em Porto Nacional.

\begin{tabular}{|l|l|}
\hline Meses & MÉDIA DO NÚMERO MÁXIMO DE DIAS SEM CHUVA \\
\hline JANEIRO & 4,2 \\
\hline FEVEREIRO & 4 \\
\hline MARÇO & 3 \\
\hline ABRIL & 6,5 \\
\hline MAIO & 12,8 \\
\hline JUNHO & 27,1 \\
\hline JULHO & 28,9 \\
\hline AGOSTO & 27,8 \\
\hline SETEMBRO & 21,4 \\
\hline OUTUBRO & 7,8 \\
\hline NOVEMBRO & 3,8 \\
\hline DEZEMBRO & 4,8 \\
\hline
\end{tabular}

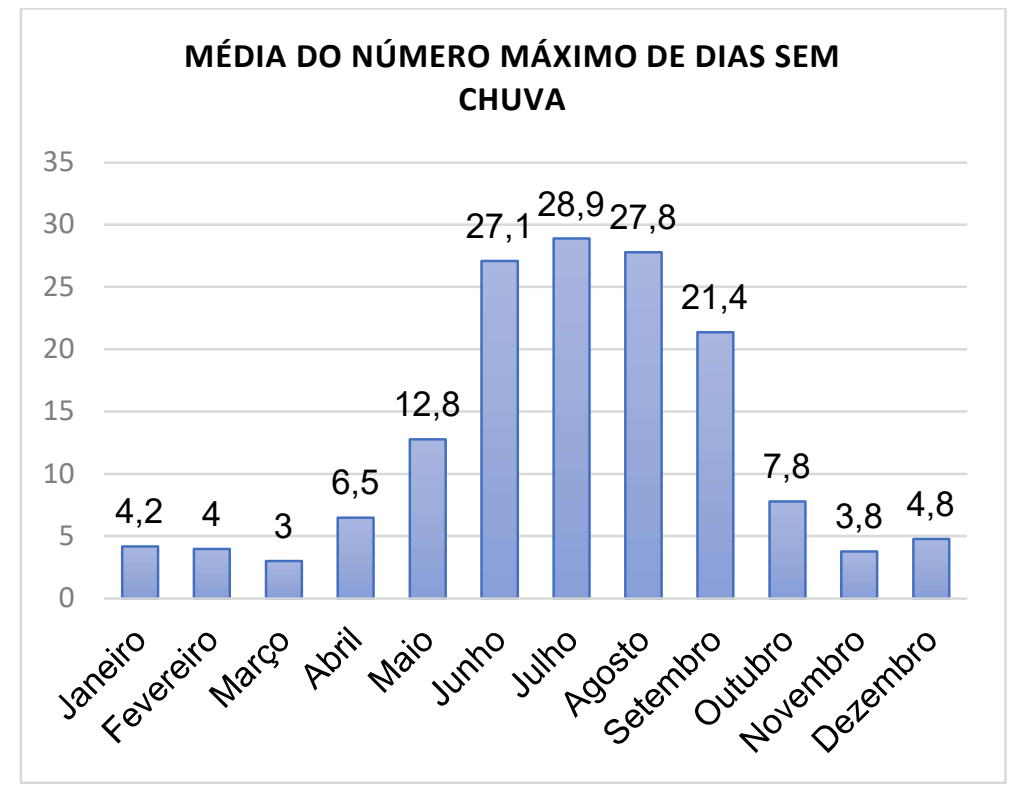

Figura 3: Média do número de dias sem chuva por mês.

Verifica-se que o mês de março é o que possui a menor média do número máximo de dias sem chuva, com 3 dias. E o mês de julho é o que possui a maior média, com 28,9, o que significa quase todo o mês sem chuva. Desse modo, a série de Máximos Dias Sem Chuva (MDS) = 28,9 dias. Segundo a ABNT NBR 15527:2007, a incógnita T para o cálculo do Método de Azevedo Neto refere-se ao valor do número de meses de seca ou de pouca chuva. No entanto a norma não especifica o modo de adoção desse número. Giacchini et al. (2011) e Lima et al. (2015) utilizaram esse T de acordo com a representatividade do maio máximo de duas sem chuva por mês. Nesse sentido, para o estudo em questão, ao considerar que o mês de julho possui 31 dias, o período seco representa 93,22\% do mês. Logo, $T=0,9322$.

Ao considerar a implantação do sistema de coleta e armazenagem de águas pluviais, identifica-se que ele seria mais necessário para os blocos Gamma ou Beta, visto que eles possuem a maior concentração de água ou acadêmicos, principalmente o bloco Beta que possui 17 salas. Para o cálculo do reservatório por meio do Método de Azevedo Neto, é necessário dispor do dado relacionado à área de captação. Nesse sentido, considerou-se o bloco Beta para tal, com todo o seu telhado. A partir da planta baixa disponibilizada pela instituição, verificou-se que o bloco beta possui uma cobertura de aproximadamente $2.202,9 \mathrm{~m}^{2}$. O valor é aproximado em virtude de o projeto disponibilizado possuir apenas a planta baixa, desse modo a área foi calculada de acordo com a planta baixa do bloco, pela falta da planta de cobertura. A partir dos dados 
verificados é possui encontrar o resultado do Método Azevedo Neto, onde:

$V=0,042 \times P \times A \times T$

$P=1572,6 \mathrm{~mm}$ ou $1,5726 \mathrm{~m}$

$A=2.202,9 \mathrm{~m}^{2}$

$\mathrm{T}=0,9322$

$V=0,042 \times 1,5726 \times 2.202,9 \times 0,9322$

$\mathrm{V}=135,63 \mathrm{~m}^{3}$

A partir dos estudos de Giacchini et al. (2011) e Lima et al. (2015), verifica-se que é recomendado admitir T com valores de 1, 2 ou 3. Diante disso, obtêm-se o disposto no quadro 3:

Quadro 3: Resultado do Método de Azevedo Neto.

\begin{tabular}{|l|l|l|l|}
\hline Precipitação $(\mathrm{m})$ & Área de Captação $(\mathrm{mm})$ & $\mathrm{T}(\mathrm{mês})$ & $\mathrm{V}\left(\mathrm{m}^{3}\right)$ \\
\hline 1,5726 & $2.202,90$ & 0,9322 & 135,63 \\
\hline 1,5726 & $2.202,90$ & 1 & 145,5 \\
\hline 1,5726 & $2.202,90$ & 2 & 291 \\
\hline 1,5726 & $2.202,90$ & 3 & 436,5 \\
\hline
\end{tabular}

Diante do quadro 3, identifica-se a necessidade da verificação dos períodos de seca, visto que o arredondamento utilizado conforme outros estudos, promove resultados muito variáveis. O reservatório calculado, considerando os dias de secas verificados, é de $135,63 \mathrm{~m}^{3}$ ou 135.630 litros. É um reservatório grande, porém suas vantagens são consideráveis, especialmente a vantagem ambiental.

\section{CONCLUSÕES}

A partir do estudo realizado, verifica-se que Porto Nacional - TO é uma cidade com um índice pluviométrico considerável, especialmente entre os meses de outubro a abril. Porém há um período de seca crítico, consistindo principalmente no mês de julho. Para a implantação de um sistema de coleta e armazenagem de águas pluviais no ITPAC, a questão da seca não se apresenta como um fator limitante, visto que o período de seca compreende o período de férias da instituição.

Apesar de o ITPAC dispor de poço artesiano e fazer o uso de água a partir de caminhões pipas, a implantação do sistema de armazenagem de águas pluviais é um método a ser considerado, visto que, além de ser sustentável, contribui para o ensino para a comunidade acadêmica de engenharia civil e arquitetura. Além disso, a implantação do método pode ser uma estratégia de ensino para os cursos, a partir da inclusão dos alunos no projeto dele. Verificou-se que, de acordo com as características do loca, é necessário um reservatório de 135.630 litros, sendo um tamanho bastante considerável. Desse modo, trata-se de um projeto de grande porte a ser implantado.

\section{REFERÊNCIAS}

AZEVÊDO, B. M. L.. Avaliação do uso da água no centro de tecnologia da UFPB. João Pessoa: Universidade Federal da Paraíba, 2016.

ABNT. Associação Brasileira das Normas Técnicas. NBR 15527: Água de chuva: Aproveitamento de coberturas em áreas urbanas para fins não potáveis: Requisitos. Rio de Janeiro, 2007.
GIACCHINI, M.; ANDRADE FILHO, A. G.; SANTOS, D. C.. Estudo do método de Azevedo Neto para dimensionamento de reservatório de água da chuva. In: SIMPÓSIO BRASILEIRO DE RECURSOS HÍDRICOS, 19. Anais. 2011.

LIMA, M. G. M.; MELO, D. F.; OLIVEIRA, H.; SOUZA, F. G.; DANTAS NETO, J.. Dimensionamento de reservatório de água 
pluvial: uso do método de Azevedo Neto para residência unifamiliar. In: WORKSHOP INTERNACIONAL SOBRE ÁGUA DO SEMIÁRIDO BRASILEIRO, 2. Anais. 2015.

MARINOSKI, A. K.. Aproveitamento de água pluvial para fins não potáveis em instituição de ensino: estudo de caso em Florianópolis - SC. Florianópolis: Universidade Federal de Santa Catarina, 2007.

MAY, S.. Estudo da viabilidade do aproveitamento de água de chuva para consumo não potável em edificações. São Paulo, 2004.
NAZÁRIO, S. L. S.; MOLINA, N. S.; PIRES, R. G.; ROCHA, D. P.. Estudo da viabilidade do aproveitamento de água pluvial no Estado de Rondônia. In: CONGRESSO NACIONAL DE EXCELÊNCIA EM GESTÃO, 9. Anais. 2013.

SCHNEIDER, L.; BARBISAN, A. O.; BENETTI, J. E.. Estudo de viabilidade de aproveitamento de águas pluviais no Centro Politécnico da UCEFF Faculdades. Tecnológica, v.4, n.1, 2016.

SODRÉ, V.. Um retrato da gestão da água no Brasil. Brasília: AESBE, 2018.

A CBPC - Companhia Brasileira de Produção Científica (CNPJ: 11.221.422/0001-03) detém os direitos materiais desta publicação. Os direitos referem-se à publicação do trabalho em qualquer parte do mundo, incluindo os direitos às renovaç̃̃es, expansões e disseminações da contribuição, bem como outros direitos subsidiários. Todos os trabalhos publicados eletronicamente poderão posteriormente ser publicados em coletâneas impressas sob coordenação da Cognitionis Publishing, da Companhia Brasileira de Produção Científica e seus parceiros autorizados. Os (as) autores (as) preservam os direitos autorais, mas não têm permissão para a publicação da contribuição em outro meio, impresso ou digital, em português ou em tradução. 